proteins and eventually began to express an added gene (GFP) linked to a Purkinje promoter. This suggests that genes could be introduced into existing damaged or unhealthy Purkinje neurons using bone marrow cells as a vehicle. As bone marrow cells can enter the brain after peripheral injection, the prospect of using such engineered cells instead of brain-derived neural stem cells, which are more difficult to harvest and need to be implanted directly in the brain, holds considerable promise.

It is tempting to speculate that fusion with bone-marrow-derived cells is a natural occurrence in the Purkinje cell population, and not one linked to irradiation or the bone marrow transplant. So far, there is no direct evidence to support this claim. Weimann and colleagues have not examined Purkinje cells in intact animals to determine whether binucleated cells exist normally. This type of analysis would be time intensive but worthwhile, as it will determine whether blood-borne cells may normally participate in Purkinje cell function. If Purkinje neurons are fusion targets for bonemarrow-derived stem cells under normal circumstances, then stem cells may provide useful therapeutic vehicles under conditions that do not require potentially lethal irradiation. $\square$
1. Alvarez-Buylla, A., Garcia-Verdugo, J., Tramontin, A. Nature Rev. Neurosci. 2, 287-293 (2001).

2. Gould, E. \& Gross, G. J. Neurosci. 22, 619-623 (2002).

3. McKay, R. Nature 406, 361-364 (2000).

4. Priller, J. et al. J. Cell Biol. 155, 733-738 (2001).

5. Brazelton, T. et al. Science 290, 1775-1779 (2000).

6. Mezey, E. et al. Science 290, 1779-1782 (2000).

7. Weimann, J. et al. Proc Natl Acad. Sci. USA 100, 2088-2093 (2003)

8. Wang, X. et al. Nature 422, 897-901 (2003)

9. Weimann, J., Johansson, C., Trejo, A. \& Blau, H. Nature Cell Biol. 5, 959-966 (2003).

10. Alvarez-Dolado, M. et al. Nature (in the press)

11. Fonnum, F. \& Lock, E. Toxicol. Lett. 112-113, 9-16 (2000).

12. Kern, J. Brain Dev. 25, 377-382 (2003).

13. Jellinger, K., Heiss, W. \& Deisenhammer, E. J. Neurol. 207, 289-305 (1974).

14. Klockgether, T. \& Evert, B. Trends Neurosci. 21, 413-418 (1998).

\section{Plant microtubule nucleation sites: moving right along}

In real estate, the common wisdom is that the key factors are location, location, location. Similarly, in the interphase plant cell the location of microtubules at the cell cortex is one key to successful management of cell growth. Unlike mitotic spindle poles, which are more readily recognizable as microtubule-nucleating sites, the location, nature and behaviour of nucleating sites for the extensive interphase cortical array have been a source of much debate over past decades.

In the average interphase plant cell, the approximately parallel allignment of most cortical microtubules makes it difficult to imagine a typical centrosomal-like nucleating site from which microtubules radiate, and thus the idea of a dispersed centrosome arose. On page 967 of this issue, Chan et al. use the microtubule end-binding protein AtEBla coupled to green fluorescent protein (GFP) to identify the long-elusive sites of microtubule nucleation. AtEB1-GFP marks both the fast-growing plus end of Arabidopsis thaliana microtubules, as well as the minus end, which in other organisms is known to be embedded in the microtubule nucleation material of spindle pole bodies and centrosomes. Although both ends of a microtubule may be aglow with GFP, they are distinguishable from each other. Using this marker, Chan et al. were able to affirm that microtubule nucleation (marked by, but not necessarily caused by, AtEB1) can occur simultaneously at numerous sites in the plant cell cortex, with each microtubule essentially arising separately. It is noteworthy that, in the suspension cells used for these experiments, microtubules of opposite polarity can be found in the same region. This suggests that the arrays of more strictly co-aligned cortical microtubules found in planta may similarly be composed of mixtures of antiparallel microtubules.

Dynamic instability of microtubules and the remodelling of cortical arrays over time must be considered when asking how microtubules come to be aligned in a particular way. As an array takes on a new orientation, do microtubules of the old orientation simply shrink and disappear at the expense of new microtubules? Although dynamic instability is undoubtedly involved in reorganizing cortical arrays, Chan et al. demonstrate that there is a

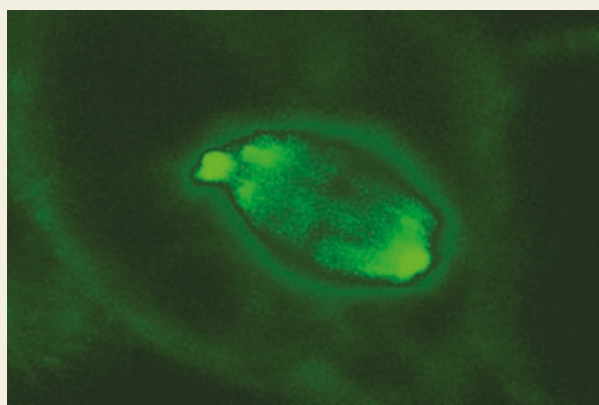

Live suspension cells of $A$. thaliana expressing AtEB1-GFP, which marks sites of microtubule nucleation. AtEB1-GFP concentrates at the spindle poles during mitosis. Scale: $3 \mathrm{~cm}$ represents $10 \mu \mathrm{m}$. Image courtesy of J. Chan and C. Lloyd.

considerable amount of 'rowdy' behaviour on the part of cortical microtubules that also contributes to microtubules re-alignment: microtubules undergoing polymerization and depolymerization can interact with their neighbours by pushing, pulling and sliding relative to them.

Much data indicates that the way plants regulate the location and orientation of interphase microtubules is fundamentally different from the situation in other kingdoms. The work of Chan et al. further expands on these differences: in place of a single centrosome from which microtubules of uniform polarity are spun out, plant cells contain multiple nucleation sites (perhaps one per microtubule?); microtubules nucleated at adjacent sites can have different polarities; and the nucleation sites, with or without microtubules attached to them, can move into and out of the cortex and to different locations within the cortex. Vive la difference!

SUE WICK

Sue Wick is in the Department of Plant Biology, University of Minnesota, St.Paul, MN55108,USA.e-mail: swick@cbs.umn.edu 